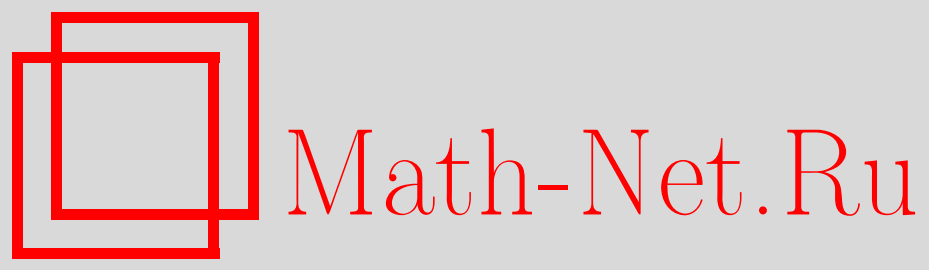

Ю. А. Неретин, Переполненные базисы в пространстве симметрических функций, Функи. анализ и его прил., 1998, том 32, выпуск 1, 12-28

DOI: https://doi.org/10.4213/faa394

Использование Общероссийского математического портала MathNet.Ru подразумевает, что вы прочитали и согласны с пользовательским соглашением

http://www . mathnet.ru/rus/agreement

Параметры загрузки:

IP : 54.205 .225 .156

26 апреля 2023 г., 06:59:41

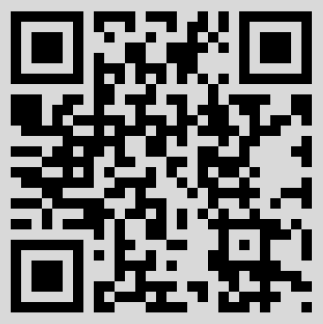


Функииональньй анализ и его приложения

1998, т. 32, вып. 1, с. 12-28

УДК 517.9

\section{Переполненные базисы в пространстве симметрических функций ${ }^{\star}$}

(c) 1998. Ю. А. НеРетин

Как известно, в теории симметрических функций важное значение имеют различные скалярные произведения (см. [3]). В книге Макдональда [3, 2nd ed.] рассматривается двупараметрическое семейство скалярных произведений (оно включает в себя в качестве частных случаев классическое скалярное произведение, скалярные произведения Холла-Литтлвуда и скалярное произведение Джека). Более общее семейство скалярных произведений, зависящее от счетного множества параметров, было введено Керовым в [2]. Во всех этих случаях произведения $p_{\lambda}$ сумм Ньютона образуют ортогональный базис, а различные скалярные произведения этого вида отличаются друг от друга нормировкой базиса $p_{\lambda}$.

В [6] было замечено, что в связи с мерами Пуассона в пространстве симметрических функций возникают скалярные произведения, отличные от керовских. В этом случае функции $p_{\lambda}$ уже не ортогональны, а простейший ортогональный (ненормированный) базис образуют мономиальные симметрические функции $m_{\lambda}$ (симметризованные одночлены).

Во всех этих случаях существует естественный унитарный изоморфизм между пространством Symm симметрических функций и бозонным пространством Фока (обсуждение этого изоморфизма в случае классического скалярного произведения см. в [8]). Естественно возникает вопрос о переносе естественных структур бозонного пространства Фока в пространство Symm и наоборот.

Основная цель статьи - выяснить, как выглядят образы гауссовых векторов бозонного пространства Фока в пространстве Symm; ответ на этот вопрос дается теоремой 1 в случае скалярных произведений Керова и формулой (21) в ситуации, связанной с пуассоновскими мерами.

На самом деле мы решаем более обшую задачу и рассматриваем семейство скалярных произведений $\langle\cdot, \cdot\rangle_{K, \omega}$ в Symm, содержащее все упомянутые выше. Эти скалярные произведения параметризуются последовательностью положительных чисел $\omega=\left(\omega_{1}, \omega_{2}, \ldots\right)$ и формальным рядом $K(h)=$ $1+\sum_{j>0} \varkappa_{j} h^{j}$ и определяются следующим образом. Пусть

$$
\Psi_{\alpha}\left(x_{1}, x_{2}, \ldots\right)=\prod_{j} K\left(\sum_{k>0} \alpha_{k} x_{j}^{k}\right) .
$$

* Работа выполнена при поддержке РФФИ и Российской программы поддержки ведущих научных школ (грант 96-01-96249). 
Тогда мы полагаем

$$
\left\langle\Psi_{\alpha}, \Psi_{\beta}\right\rangle_{K, \omega}=\exp \left(\sum_{k>0} \alpha_{j} \overline{\beta_{j}} \omega_{j}\right)
$$

и этим равенством скалярное произведение в Symm определяется однозначно. Керовские произведения получаются в случае $K(h)=\exp (h)$ (и, в частности, классический случай соответствует $\omega_{j}=j$ ), с мерами Пуассона связан случай $K(h)=1+h$.

В этой более общей ситуации формула для гауссовых векторов дается теоремами 2, 2'. Таким образом, мы получаем в пространстве Symm, точнее в его гильбертовом пополнении, некоторые переполненные базисы, состоящие из гауссовых векторов. Напомним, что под переполненным базисом подразумевается тотальное семейство векторов гильбертова пространства, голоморфно зависящее от параметра (пробегаюшего некоторое комплексное многообразие), такое, что попарные скалярные произведения задаются явной формулой. Известно, что анализ в гильбертовых пространствах часто бывает удобно строить, опираясь на переполненные базисы (или, что то же самое, «системы когерентных состояний»), а не на обычные ортогональные базисы.

Встает вопрос о том, как разложить наши переполненные базисы по различным стандартным базисам в Symm. Ввиду многочисленности стандартных базисов (см. [3]) мы приводим лишь некоторые из этих разложений (теорема 3 ).

Наконец, в 3 обсуждается пространство АSymm кососимметрических функций и вопрос о переносе фермионных гауссовых векторов в пространства ASymm и Symm.

\section{§1. Мультипликативные бозон-симметрические соответствия}

1.1. Пространства $l_{2}(\omega)$ и $l_{2}^{\circ}(\omega)$. Фиксируем последовательность

$$
\omega=\left(\omega_{1}, \omega_{2}, \ldots\right)
$$

положительных чисел. Через $l_{2}(\omega)$ мы обозначим пространство последовательностей $\alpha=\left(\alpha_{1}, \alpha_{2}, \ldots\right)$, удовлетворяющих условию $\sum_{j}\left|\alpha_{j}\right|^{2} \omega_{j}<\infty$. Скалярное произведение в $l_{2}(\omega)$ задается формулой

$$
\langle\alpha, \beta\rangle=\sum_{j=1}^{\infty} \alpha_{j} \overline{\beta_{j}} \omega_{j}
$$

Через $l_{2}^{\circ}(\omega)$ обозначим пространство последовательностей $\alpha=\left(\alpha_{1}, \alpha_{2}, \ldots\right)$, удовлетворяющих условию $\sum_{j}\left|\alpha_{j}\right|^{2} / \omega_{j}<\infty$. Скалярное произведение в $l_{2}^{\circ}(\omega)$ задается формулой

$$
\langle\alpha, \beta\rangle=\sum_{j=1}^{\infty} \frac{\alpha_{j} \overline{\beta_{j}}}{\omega_{j}} .
$$


Естественно считать пространство $l_{2}^{\circ}(\omega)$ двойственным пространством к $l_{2}(\omega)$, причем спаривание $l_{2}^{\circ}(\omega) \times l_{2}(\omega) \rightarrow \mathbb{C}$ задается формулой $\{\alpha, \beta\}=$ $\sum \alpha_{j} \beta_{j}$.

1.2. Пространства $\boldsymbol{H}^{2}(\omega)$. Пусть последовательность $\omega$ имеет не более чем экспоненциальный рост, т.е. существуют $C$ и $\sigma$, такие, что $\omega_{j} \leqslant$ $C \exp (\sigma j)$ для всех $j$. Пусть $\alpha \in l_{2}(\omega)$. Тогда ряд

$$
f(z)=\sum_{j>0} \alpha_{j} z^{j}
$$

сходится в круге $|z|<\rho:=\exp (-\sigma)$. При этом $f(0)=0$. Таким образом, мы отождествляем пространство $l^{2}(\omega)$ с гильбертовым пространством функций вида (1), голоморфных в круге $|z|<\rho$, со скалярным произведением

$$
\left\langle\sum_{j>0} \alpha_{j} z^{j}, \sum_{j>0} \beta_{j} z^{j}\right\rangle=\sum_{j>0} \alpha_{j} \overline{\beta_{j}} \omega_{j} .
$$

Это пространство мы обозначим через $H^{2}(\omega)$.

Воспроизводящее ядро пространства $H^{2}(\omega)$ задается формулой

$$
K(z, u)=\sum_{j>0} \frac{z^{j} \bar{u}^{j}}{\omega_{j}} .
$$

Последнее означает, что для любой функции $f \in H^{2}(\omega)$ и любого $а$ из круга сходимости выполнено воспроизводящее свойство:

$$
\langle f(z), K(z, a)\rangle_{H^{2}(\omega)}=f(a) .
$$

ПримеР 1. Пусть $\omega_{j}=j$. Тогда

$$
\langle f(z), g(z)\rangle=\frac{i}{2 \pi} \iint_{|z|<1} f^{\prime}(z) \overline{g^{\prime}(z)} d z d \bar{z},
$$

a $K(z, u)=-\ln (1-z \bar{u})$.

Пример 2. Пусть $\omega_{j}=j\left(1-q^{j}\right) /\left(1-t^{j}\right)$, где $q, t$ фиксированы, $0<q<1$, $0<t<1$. Тогда скалярное произведение в $H^{2}(\omega)$ задается формулой

$$
\langle f(z), g(z)\rangle=\frac{i}{2 \pi} \sum_{k=0}^{\infty} \iint_{q t^{k}<|z|^{2}<t^{k}} f^{\prime}(z) \overline{g^{\prime}(z)} d z d \bar{z},
$$

а воспроизводящее ядро пространства $H^{2}(\omega)$ равно

$$
K(z, u)=\ln \left[\prod_{n \geqslant 0} \frac{1-z \bar{u} t q^{n}}{1-z \bar{u} q^{n}}\right] .
$$

Формула (3) для скалярного произведения в пространстве голоморфных функций выглядит несколько необычно. См., однако, в работе [7] о скалярных произведениях похожего типа. 
1.3. Бозонное пространство Фока. Пусть $H-$ гильбертово пространство. Бозонное пространство Фока $F(H)$ - это гильбертово пространство, в котором фиксирована система векторов («переполненный базис») $\Phi_{h}$, нумеруемая векторами $h \in H$, такая, что

a) $\left\langle\Phi_{h}, \Phi_{h^{\prime}}\right\rangle_{F(H)}=\exp \left(\left\langle h^{\prime}, h\right\rangle_{H}\right)$;

б) линейная оболочка векторов $\Phi_{h}$ плотна в $F(H)$.

Каждому вектору $q \in F\left(l_{2}(\omega)\right)$ мы поставим в соответствие функцию $f=f_{q}$ на $l_{2}^{\circ}(\omega)$, голоморфно зависящую от $z=\left(z_{1}, z_{2}, \ldots\right)$, по формуле

$$
f_{q}\left(z_{1}, z_{2}, \ldots\right)=\left\langle q, \Phi_{\left(z_{1} / \omega_{1}, z_{2} / \omega_{2}, \ldots\right)}\right\rangle_{F\left(l_{2}(\omega)\right)} .
$$

Например, элементу переполненного базиса $\Phi_{\left(a_{1}, a_{2}, \ldots\right)}$ соответствует функция $\exp \left(\sum z_{j} \overline{a_{j}}\right)$.

Мы будем отождествлять бозонное пространство Фока $F\left(l_{2}(\omega)\right)$ с пространством голоморфных функций $f_{q}\left(z_{1}, z_{2}, \ldots\right)$ на $l_{2}^{\circ}(\omega)$.

Функции $z_{1}^{k_{1}} z_{2}^{k_{2}} \ldots$ образуют ортогональный базис в $F\left(l_{2}(\omega)\right)$ и

$$
\left\|z_{1}^{k_{1}} z_{2}^{k_{2}} \ldots\right\|^{2}=\prod\left(\omega_{j}^{k_{j}} \cdot k_{j} !\right) .
$$

\section{4. Пространство симметрических функций. Пусть $x_{1}, x_{2}, \ldots$} счетный набор формальных переменных. Симметрический формальный ряд — это формальный ряд от переменных $x_{1}, x_{2}, \ldots$, не меняющийся при любых перестановках переменных. Симметрический многочлен («симметрическая функция» в смысле [3]) — это симметрический формальный ряд, такой, что степень входящих в него одночленов $x_{1}^{k_{1}} x_{2}^{k_{2}} \ldots$ ограничена; отметим, что число слагаемых в (отличном от константы) симметрическом многочлене бесконечно. Пространство симметрических многочленов мы будем обозначать через Symm или, если нам будет важно подчеркнуть, что рассматриваются многочлены, симметричные по переменным $x_{1}, x_{2}, \ldots$, через $\operatorname{Symm}(x)$.

Напомним стандартные обозначения (см. [3, §I.2]):

$$
p_{k}=p_{k}(x)=x_{1}^{k}+x_{2}^{k}+\ldots, \quad m_{\lambda}(x)=m_{\lambda_{1} \cdots \lambda_{s}}(x)=\sum x_{j_{1}}^{\lambda_{1}} \cdots x_{j_{s}}^{\lambda_{s}},
$$

где $\lambda_{j}>0$. В последней сумме берется одночлен $x_{1}^{\lambda_{1}} x_{2}^{\lambda_{2}} \ldots$ и к нему прибавляются все (различные) одночлены, которые могут быть получены из него перестановками сомножителей. Удобно считать, что $\lambda_{1} \geqslant \lambda_{2} \geqslant \ldots$

1.5. Бозон-симметрические соответствия. Фиксируем последовательность $\omega=\left(\omega_{1}, \omega_{2}, \ldots\right)$ и, следуя Керову [2], вводим в пространстве Symm скалярное произведение $\langle\cdot, \cdot\rangle_{\omega}$ с помощью условия, что векторы $p_{1}^{k_{1}} \cdots p_{\alpha}^{k_{\alpha}}$ образуют ортогональный базис, причем

$$
\left\|p_{1}^{k_{1}} \cdots p_{\alpha}^{k_{\alpha}}\right\|_{\omega}^{2}=\prod k_{j} ! \omega_{j}^{k_{j}} .
$$

ЗАмЕЧАНИЕ. а) Классическое скалярное произведение в Symm (см. [3, § I.4]) соответствует случаю последовательности $\omega_{j}=j$.

б) О скалярных произведениях, соответствующих $\omega_{j}=j\left(1-q^{j}\right) /\left(1-t^{j}\right)$ (п. 1.2 выше), см. [3, $\S$ III.5, VI.6]. 
Рассмотрим пополнение $\mathrm{Symm}_{\omega}$ пространства Symm по норме, определенной скалярным произведением $\langle\cdot, \cdot\rangle_{\omega}$. Рассмотрим унитарный изоморфизм $I: F\left(l_{2}(\omega)\right) \rightarrow$ Symm $_{\omega}$, ставящий в соответствие любой голоморфной функции $f\left(z_{1}, z_{2}, \ldots\right) \in F\left(l_{2}(\omega)\right)$ (где $\left.z=\left(z_{1}, z_{2}, \ldots\right) \in l_{2}^{\circ}(\omega)\right)$ формальный симметрический ряд $f\left(p_{1}(x), p_{2}(x), \ldots\right)$. Иными словами, мы просто делаем подстановку $z_{j}=p_{j}(x)$ (в силу формул (6)-(7) этот оператор действительно унитарен).

Например, элементу переполненного базиса

$$
\exp \left(\sum \alpha_{n} z_{n}\right) \in F\left(l_{2}(\omega)\right)
$$

соответствует симметрический формальный ряд

$$
\exp \left(\sum \alpha_{n} p_{n}(x)\right)=\prod_{j} \exp \left(\sum_{n} \alpha_{n} x_{j}^{n}\right) \in \operatorname{Symm}_{\omega} .
$$

1.6. Гауссовы векторы. Переполненный базис $\Phi_{h} \in F\left(l_{2}(\omega)\right)$ включается в более широкий переполненный базис, состоящий из гауссовых векторов $b[Q \mid r]$. Эти векторы (функции на $\left.l_{2}^{\circ}(\omega)\right)$ задаются формулой

$$
\exp \left\{\frac{1}{2} \sum q_{i j} z_{i} z_{j}+\sum r_{j} z_{j}\right\} \in F\left(l_{2}(\omega)\right),
$$

где $q_{i j}=q_{j i}$. Обозначим через $\Omega$ диагональную матрицу, у которой на диагонали стоят числа $\omega_{1}, \omega_{2}, \ldots$ Вектор $b[Q \mid r]$ содержится в $F\left(l_{2}(\Omega)\right)$ в том и только том случае, когда выполнены следующие условия:

(i) $r \in l_{2}^{\circ}(\omega)$;

(ii) матрица $R:=\Omega^{-1 / 2} Q \Omega^{-1 / 2}$ является матрицей Гильберта-Шмидта (т.е. след матрицы $R^{*} R$ конечен);

(iii) норма матрицы $R$ (в смысле нормы оператора в (обычном) $l_{2}$ ) меньше единицы.

Скалярные произведения векторов $b[Q \mid r]$ (если они действительно лежат в $\left.F\left(l_{2}(\omega)\right)\right)$ легко вычисляются; см., например, [5, §6.2].

Впрочем, с точки зрения настоящей статьи естественнее рассматривать выражение (8) как формальный ряд и не обращать никакого внимания на упомянутые условия (i)-(iii).

Нас интересует, какие симметрические функции соответствуют гауссовым векторам $b[Q \mid r]$.

Tеорема 1. Элемент $\operatorname{Symm}_{\omega}$, соответствуюший вектору $b[Q \mid r]$, задаетсл формулой

$$
\prod_{i<j} \exp \left(\sum_{m, n>0} q_{m n} x_{i}^{m} x_{j}^{n}\right) \prod_{i} \exp \left(\sum_{n>0}\left(r_{n}+\frac{1}{2} \sum_{\alpha+\beta=n} q_{\alpha \beta}\right) x_{i}^{n}\right) .
$$

ДокАЗАтЕльство очевидно. Вектору $b[Q \mid r]$ соответствует симметрический формальный ряд

$$
\begin{aligned}
& \exp \left\{\frac{1}{2} \sum q_{m n} p_{m}(x) p_{n}(x)+\sum r_{n} p_{n}(x)\right\} \\
& =\exp \left\{\frac{1}{2} \sum q_{m n}\left(x_{1}^{m}+x_{2}^{m}+\cdots\right)\left(x_{1}^{n}+x_{2}^{n}+\cdots\right)+\sum r_{n}\left(x_{1}^{n}+x_{2}^{n}+\cdots\right)\right\} .
\end{aligned}
$$


Далее проводим очевидные преобразования.

ЗАмечАниЕ. Таким образом, гауссовым формальным рядам $b[Q \mid r]$ вида (8) соответствуют в точности всевозможные произведения вида

$$
\prod_{i>j} S\left(x_{i}, x_{j}\right) \cdot \prod_{i} T\left(x_{i}\right),
$$

где формальные ряды $S$ и $T$ имеют вид

$$
S(x, y)=1+\sum_{m>0, n>0} s_{m n} x^{m} y^{n}, \quad s_{m n}=s_{n m}, \quad T(x)=1+\sum_{n>0} t_{n} x^{n} .
$$

\section{7. Пример: алгебра Вирасоро и образы гауссовых векторов.} Пусть $\omega_{j}=j$. Фиксируем $\alpha, \beta \in \mathbb{C}$. Рассмотрим представления алгебры Вирасоро в пространстве Фока $F\left(H^{2}(\omega)\right)$ операторами $L_{n}$ (где $\left.n \in \mathbb{Z}\right)$, заданными стандартными формулами (см., например, $[4,5])$ :

$$
\begin{gathered}
L_{n}=\sum_{k \geqslant 0} z_{n+k} k \frac{\partial}{\partial z_{k}}+\frac{1}{2} \sum_{\substack{k+m=n \\
k>0, m>0}} z_{m} z_{k}+(\alpha+i \beta n) z_{n} \quad \text { при } n>0, \\
L_{k}:=L_{-k}^{*} \quad \text { при } k<0, \quad L_{0}=\sum z_{k} k \frac{\partial}{\partial z_{k}}+\frac{1}{2}\left(\alpha^{2}+\beta^{2}\right) .
\end{gathered}
$$

Тогда для всех $m, n$ выполнено равенство

$$
\left[L_{m}, L_{n}\right]=(n-m) L_{m+n}+\frac{n^{3}-n}{24}\left(1+12 \beta^{2}\right) \delta_{m+n, 0} .
$$

Рассмотрим проективное представление группы Diff аналитических диффеоморфизмов окружности, сохраняющих ориентацию, соответствующее представлению (9)-(10) алгебры Вирасоро (явные формулы см. в [4]). Эта группа действует в бозонном пространстве Фока, а следовательно, и в пространстве $\operatorname{Symm}_{\omega}$. Орбита вектора $f(z)=1$ бозонного пространства Фока под действием группы Diff состоит из гауссовых векторов; явные формулы для этих векторов получены в [4]. Опишем орбиту (симметрической) функции 1 под действием группы Diff. Прослеживание нашей конструкции и конструкции из $[4, \S 4]$, показывает, что орбита состоит из симметрических формальных рядов вида

$$
\Xi_{\theta}(x)=\prod_{i>j}\left(\frac{\theta\left(x_{i}\right)-\theta\left(x_{j}\right)}{x_{i}-x_{j}}\right) \cdot \prod_{i} \theta^{\prime}\left(x_{i}\right)^{1+\alpha} \cdot \prod_{i}\left(\frac{\theta\left(x_{i}\right)}{x_{i}}\right)^{\beta},
$$

где $\theta(z)$ пробегает все функции вида

$$
\theta(z)=z+\theta_{2} z^{2}+\theta_{3} z^{3}+\ldots,
$$

однолистные в круге $|z|<1+\varepsilon($ где $\varepsilon=\varepsilon(\theta)>0)$.

1.8. Операторы. Рассмотрим бозонные пространства Фока $F\left(l_{2}(\omega)\right)$ и $F\left(l_{2}(\nu)\right)$. Рассмотрим формальный ряд (ядро)

$$
L(z, u)=\sum l_{i_{1} i_{2} \ldots}^{j_{1} j_{2} \ldots} z_{1}^{i_{1}} z_{2}^{i_{2}} \ldots \bar{u}_{1}^{j_{1}} \bar{u}_{2}^{j_{2}} \ldots
$$


Положим $l_{z}(u):=\overline{L(z, u)}$. Далее определим оператор $\widehat{L}: F\left(l_{2}(\omega)\right) \rightarrow F\left(l_{2}(\nu)\right)$ по формуле

$$
\widehat{L} f(z)=\left\langle f, l_{z}\right\rangle_{F\left(l_{2}(\omega)\right)} .
$$

Напомним (см., например, $[5, \S 6.1])$, что любой ограниченный оператор из $F\left(l_{2}(\omega)\right)$ в $F\left(l_{2}(\nu)\right)$ действительно представим в таком виде.

Опишем, как задать соответствующий оператор

$$
\widehat{\mathscr{L}}: \operatorname{Symm}_{\omega}(y) \rightarrow \operatorname{Symm}_{\nu}(x) .
$$

Рассмотрим формальный ряд $\mathscr{L}\left(x_{1}, x_{2}, \ldots, y_{1}, y_{2}, \ldots\right)$ (мы будем называть его бисимметрическим ядром), симметричный по набору переменных $x_{1}$, $x_{2}, \ldots$, a также по набору переменных $y_{1}, y_{2}, \ldots$, заданный формулой

$$
\begin{aligned}
\mathscr{L}(x, y) & =L\left(p_{1}(x), p_{2}(x), \ldots ; p_{1}(y), p_{2}(y), \ldots\right) \\
& =\sum l_{i_{1} i_{2} \ldots}^{j_{1} j_{2} \ldots} p_{1}(x)^{i_{1}} p_{2}(x)^{i_{2}} \ldots p_{1}(y)^{j_{1}} p_{2}(y)^{j_{2}} \ldots .
\end{aligned}
$$

Тогда

$$
\widehat{\mathscr{L}} g(x)=\langle g, \overline{\mathscr{L}(x, y)}\rangle_{\operatorname{Symm}_{\omega}(y)} .
$$

1.9. Гауссовы операторы. Гауссов оператор $F\left(l_{2}(\omega)\right) \rightarrow F\left(l_{2}(\nu)\right)$ - это оператор с ядром вида

$$
\begin{aligned}
L(z, u) & =\exp \left\{\frac{1}{2}\left(\begin{array}{ll}
z & \bar{u}
\end{array}\right)\left(\begin{array}{cc}
K & L \\
L^{t} & M
\end{array}\right)\left(\begin{array}{c}
z^{t} \\
\bar{u}^{t}
\end{array}\right)\right\} \\
& =\exp \left\{\frac{1}{2} \sum k_{i j} z_{i} z_{j}+\sum l_{i j} z_{i} \bar{u}_{j}+\frac{1}{2} \sum m_{i j} \bar{u}_{i} \bar{u}_{j}\right\} .
\end{aligned}
$$

Соответствуюшее бисимметрическое ядро $\mathscr{L}(x, y)$ задается формулой

$$
\begin{aligned}
\prod_{\alpha>\beta} \exp \left\{\sum_{i, j} k_{i j} x_{\alpha}^{i} x_{\beta}^{j}\right\} & \cdot \prod_{\alpha, \beta} \exp \left\{\sum_{i, j} l_{i j} x_{\alpha}^{i} y_{\beta}^{j}\right\} \cdot \prod_{\alpha>\beta} \exp \left\{\sum_{i, j} m_{i j} y_{\alpha}^{i} y_{\beta}^{j}\right\} \\
& \times \prod_{\alpha} \exp \left\{\frac{1}{2} \sum_{i, j} k_{i j} x_{\alpha}^{i+j}\right\} \cdot \prod_{\alpha} \exp \left\{\frac{1}{2} \sum_{i, j} m_{i j} y_{\alpha}^{i+j}\right\} .
\end{aligned}
$$

1.10. Пример: бисимметрическое ядро единичного оператора. Ядро единичного оператора $F\left(l_{2}(\omega)\right) \rightarrow F\left(l_{2}(\omega)\right)$ задается формулой

$$
L(z, u)=\exp \left\{\sum \frac{z_{i} \bar{u}_{i}}{\omega_{i}}\right\} .
$$

Заметим, что в показателе экспоненты стоит воспроизводящее ядро пространства $H^{2}(\omega)$ (см. п. 1.2). Соответствующее бисимметрическое ядро $\mathscr{L}$ равно

$$
\mathscr{L}(x, y)=\prod_{\alpha, \beta} \exp \left\{\sum \frac{x_{\alpha}^{i} y_{\beta}^{i}}{\omega_{i}}\right\} .
$$


В частности, в классическом случае $\omega_{j}=j$ мы имеем

$$
\mathscr{L}(x, y)=\prod_{\alpha, \beta}\left(1-x_{\alpha} y_{\beta}\right)^{-1}
$$

а в случае $\omega_{j}=j\left(1-q^{j}\right) /\left(1-t^{j}\right)$ в силу (4) получаем

$$
\mathscr{L}(x, y)=\prod_{\alpha, \beta} \prod_{n \geqslant 0} \frac{1-x_{\alpha} y_{\beta} t q^{n}}{1-x_{\alpha} y_{\beta} q^{n}} .
$$

Теперь мы можем сравнить наши высказывания с рядом известных теорем. Пусть дано некоторое скалярное произведение $\langle\cdot, \cdot\rangle$ в пространстве Symm, причем подпространства однородных многочленов попарно ортогональны. Рассмотрим однородный ортонормированный базис $u_{\xi}(x)$ в пространстве Symm. Воспроизводящее ядро для скалярного произведения $\langle\cdot, \cdot\rangle$ задается формулой

$$
\sum_{\xi} u_{\xi}(x) \overline{u_{\xi}(y)}
$$

Легко видеть, что оператор в $\mathrm{Symm}_{\omega}$, соответствующий бисимметрическому ядру (14), есть в точности единичный оператор (отсюда, в частности, вытекает, что выражение (14) не зависит от выбора базиса $\left.u_{\xi}\right)$. Поэтому формулы (11)-(13) суть формулы для воспроизводящих ядер. Формула (12) — это классическая формула, см. [3, I.4]. Формула (13) - формула Макдональда [3, VI.2, 2nd ed.] (см. также [3, §III.4]; аналогично получается формула для воспроизводящего ядра (см. [3, VI.10]) в случае многочленов Джека). Формула (11) обнаружена Керовым [2].

\section{§2. Немультипликативные бозон-симметрические соответствия}

2.1. Базисы $\mathfrak{q}(\boldsymbol{x})$. Фиксируем формальный ряд

$$
K(h)=1+\sum_{j>0} \varkappa_{j} h^{j}
$$

где $\varkappa_{1} \neq 0$. Рассмотрим симметрический формальный ряд

$$
\Psi_{\alpha}(x)=\prod_{\xi} K\left(\sum_{j>0} \alpha_{j} x_{\xi}^{j}\right)
$$

где произведение берется по всем переменным $x_{\xi}=x_{1}, x_{2}, \ldots$.

Разложим выражение $\Psi_{\alpha}$ в ряд по переменным $\alpha_{1}, \alpha_{2}, \ldots$ :

$$
\Psi_{\alpha}(x)=\sum_{n_{1}, n_{2}, \ldots} \alpha_{1}^{n_{1}} \alpha_{2}^{n_{2}} \ldots \mathfrak{q}_{n_{1}, n_{2}, \ldots}(x) .
$$

Легко видеть, что симметрические многочлены $\mathfrak{q}_{n_{1}, n_{2}, \ldots}(x)=\mathfrak{q}_{n_{1}, n_{2}, \ldots}^{K}(x)$ образуют однородный базис в Symm . 
ПРИмЕР. Если $K(h)=\exp (h)$, то

$$
\mathfrak{q}_{n_{1}, n_{2}, \ldots}(x)=\prod \frac{p_{j}^{n_{j}}}{n_{j} !} .
$$

Если $K(h)=1+h$ (см. ниже п. 2.3), то базис q состоит из полных симметрических функций $m_{\lambda}$, см. п. 1.4 .

2.2. Бозон-симметрические соответствия. Фиксируем формальный ряд $K(x)$ (см. (15)) и последовательность $\omega$ (см. п. 1.1). Определим скалярное произведение $\langle\cdot, \cdot\rangle_{K, \omega}$ в Symm с помощью условия, что многочлены $\mathfrak{q}_{n_{1}, n_{2}, \ldots}^{K}(x)$ образуют ортогональный базис и

$$
\left\|\mathfrak{q}_{n_{1}, n_{2}, \ldots}^{K}\right\|^{2}=\prod \frac{\omega_{j}^{n_{j}}}{n_{j} !} .
$$

Дадим также равносильное, но более удобное определение скалярного произведения $\langle\cdot, \cdot\rangle_{K, \omega}$, задав его на элементах переполненного базиса $\Psi_{\alpha}$ :

$$
\left\langle\Psi_{\alpha}, \Psi_{\beta}\right\rangle_{K, \omega}=\left\langle\prod_{\xi} K\left(\sum_{j>0} \alpha_{j} x_{\xi}^{j}\right), \prod_{\xi} K\left(\sum_{j>0} \beta_{j} x_{\xi}^{j}\right)\right\rangle_{K, \omega}=\exp \left(\sum \alpha_{j} \bar{\beta}_{j} \omega_{j}\right) .
$$

Обозначим через $\mathrm{Symm}_{K, \omega}$ пополнение пространства Symm по этому скалярному произведению (очевидно, что элементы из $\mathrm{Symm}_{K, \omega}$ реализуются как формальные симметрические ряды).

Унитарный изоморфизм между $F\left(l_{2}(\omega)\right)$ и $\operatorname{Symm}_{K, \omega}$ мы установим следующим образом: элементу $\Phi_{\alpha} \in F\left(l_{2}(\omega)\right)$ (см. п. 1.3) поставим в соответствие элемент $\Psi_{\alpha} \in \operatorname{Symm}_{K, \omega}$. В силу условия а) п. 1.3 и формулы (17) это соответствие действительно определяет унитарный оператор.

Рассмотрим произвольную функцию

$$
f\left(z_{1}, z_{2}, \ldots\right)=\sum c_{n_{1}, n_{2}, \ldots} \prod \frac{z_{j}^{n_{j}}}{n_{j} !}
$$

из $F\left(l_{2}(\omega)\right)$. Легко видеть, что ей соответствует симметрический формальный ряд

$$
\mathscr{F}(x)=\sum c_{n_{1}, n_{2}, \ldots} \mathfrak{q}_{n_{1}, n_{2}, \ldots}^{K}(x) .
$$

2.3. Пример: $K(h)=1+h$ (см. [6]). Рассмотрим меру $\mu$ на $\mathbb{C}^{*}=\mathbb{C} \backslash 0$, инвариантную относительно вращений. Пусть $\mu$ удовлетворяет следующему условию: все величины

$$
\omega_{j}=\int_{\mathbb{C}^{*}}|z|^{2 j} d \mu(z), \quad j=1,2, \ldots,
$$

конечны. В частности, мера любого диска $|z| \geqslant \varepsilon$ конечна (однако мера всего $\mathbb{C}^{*}$ может быть бесконечной).

Обозначим через $\Omega$ множество всех конечных или счетных подмножеств в $\mathbb{C}^{*}$. Введем на $\Omega$ меру Пуассона $\nu$. Напомним ее определение. Пусть 
$M_{1}, \ldots, M_{k}$ - попарно не пересекающиеся подмножества конечной меры в $\mathbb{C}^{*}$. Тогда вероятность того, что $M_{j}$ содержит ровно $p_{j}$ точек (для всех $j$ ) равна

$$
\prod_{j}\left[\frac{\mu\left(M_{j}\right)^{p_{j}}}{p_{j} !} \exp \left(-\mu\left(M_{j}\right)\right)\right]
$$

Сформулируем это чуть-чуть иначе. Рассмотрим множество $\mathscr{A}\left[M_{1}, \ldots, M_{k}\right.$; $\left.p_{1}, \ldots, p_{k}\right]$ состоящее из всех $\zeta=\left\{\zeta_{1}, \zeta_{2}, \ldots\right\} \in \Omega$, таких, что пересечение $\zeta$ с $M_{j}$ состоит точно из $j$ точек. Тогда мера этого множества $\nu\left(\mathscr{A}\left[M_{1}, \ldots, M_{k}\right.\right.$; $\left.\left.p_{1}, \ldots, p_{k}\right]\right)$ задается формулой $(19)$. Если $\mu\left(\mathbb{C}^{*}\right)<\infty$, то мера $\nu$ сосредоточена на множестве конечных подмножеств в $\mathbb{C}^{*}$. Если $\mu\left(\mathbb{C}^{*}\right)=\infty$, то мера $\nu$ сосредоточена на пространстве последовательностей, сходящихся к 0 .

Симметрическому многочлену $f\left(x_{1}, x_{2}, \ldots\right)$ мы поставим в соответствие настоящую функцию (определенную почти всюду) на $\Omega$ по следующему правилу. Пусть $\zeta=\left\{\zeta_{1}, \zeta_{2}, \ldots\right\} \in \Omega$. Тогда наша функция получается подстановкой $x_{1}=\zeta_{1}, x_{2}=\zeta_{2}, \ldots$. Получающиеся таким образом ряды действительно сходятся в $L^{2}(\Omega, \nu)$.

Итак, пространство симметрических многочленов вкладывается в пространство $L^{2}(\Omega, \nu)$, и поэтому на пространстве Symm индуцируется некоторое скалярное произведение (отметим, что Symm не плотно в $L^{2}$ ). Оказывается, что это скалярное произведение соответствует функции $K(h)=1+h$ и последовательности $\omega_{j}$, заданной формулой $(18)$.

2.4. Образы гауссовых векторов. Под графом мы будем понимать неориентированный граф с конечным множеством ребер (множество вершин у нас будет бесконечно), причем мы допускаем кратные ребра (т.е. считаем, что из одной вершины в другую может идти несколько ребер), а также петли (т.е. ребра, ведущие из вершины в нее же). Под оснашеннылм графом мы будем понимать граф, вершины которого суть переменные $x_{1}, x_{2}, \ldots$ и каждой вершине $x_{i}$ приписано целое неотрицательное число $w_{i}$ (которое мы будем называть довеском), причем все довески $w_{i}$, кроме конечного числа, должны быть равны 0 .

Для оснащенного графа $\Gamma$ введем обозначения:

- $s_{i j}$ — число ребер, ведущих из $x_{i}$ в $x_{j}$; (в частности) $s_{i i}$ - число петель с началом и концом в $x_{i}$;

- $m_{i}$ (вес вершины) - число ребер, выходящих из $x_{i}$ плюс довесок $w_{i}$; иными словами $m_{i}=w_{i}+2 s_{i i}+\sum_{j \neq i} s_{i j}$;

- $\operatorname{edge}(\Gamma)$ - множество ребер графа $\Gamma$;

- $u(\gamma), v(\gamma)$ - концы ребра $\gamma$.

Рассмотрим теперь гауссов вектор $b[Q \mid r] \in F\left(l_{2}(\omega)\right)$. Положим

$$
\widetilde{Q}(x, y)=\sum q_{i j} x^{i} y^{j}, \quad \tilde{r}(x)=\sum r_{j} x^{j} .
$$

Tеорема 2. Вектору $b[Q \mid r] \in F\left(l_{2}(\omega)\right)$ соответствует элемент $\mathfrak{B}^{K}[Q \mid r]$ пространства $\mathrm{Symm}_{K, \omega}$, задаваемый Формулой

$\sum_{\Gamma}\left\{\frac{\prod_{i} m_{i} !}{\prod_{i>j} s_{i j} ! \cdot \prod_{i}\left[s_{i i} ! \cdot 2^{s_{i i}} \cdot w_{i} !\right]} \cdot \prod_{i} \varkappa_{m_{i}} \cdot \prod_{\gamma \in \operatorname{edge}(\Gamma)} \widetilde{Q}\left(x_{u(\gamma)}, x_{v(\gamma)}\right) \cdot \prod_{i} \tilde{r}\left(x_{i}\right)^{w_{i}}\right\}$, 
где суммирование ведется по всем оснащенным графам Г.

Факториальный коэффициент в этой формуле может быть убран с помощью лингвистического преобразования, которое и проделывается ниже в пा. 2.5-2.6.

ПримеР. Пусть $K(h)=1+h$. Обозначим через $\mathscr{R}$ множество всех разбиений натурального ряда $1,2,3, \ldots$ на двухточечные и одноточечные подмножества, и пусть при этом число двухточечных подмножеств конечно. Тогда формула (20) переписывается в виде

$$
\sum_{S \in \mathscr{R}}\left(\prod_{\{\alpha, \beta\} \in S} \widetilde{Q}\left(x_{\alpha}, x_{\beta}\right) \cdot \prod_{\{\sigma\} \in S}\left(1+\tilde{r}\left(x_{\sigma}\right)\right)\right) ;
$$

здесь суммирование ведется по всем разбиениям натурального ряда (или, что то же самое, по всем разбиениям множества переменных $x_{1}, x_{2}, \ldots$ ) указанного выше типа, $\{\alpha, \beta\}$ пробегают все двухточечные подмножества разбиения $S \in \mathscr{R}$, а $\sigma$ пробегает все одноточечные подмножества этого разбиения.

2.5. Связки. Пусть $N$ - счетное множество. Ниже $N$ будет совпадать с натуральным рядом $\mathbb{N}$ или с множеством $\mathscr{X}$ симметрических переменных $x_{1}, x_{2}, \ldots$. Поставим в соответствие каждому элементу $a \in N$ целое неотрицательное число $l_{a}$ так, что все числа $l_{a}$, кроме конечного числа, равны 0 . Такой набор данных мы назовем конфигураиией над $N$. Удобно думать, что мы выбираем из множества $N$ точки и $l_{a}$ есть число раз, которое нам попалась точка $a$. Во избежание двусмысленностей, которые могли бы возникнуть ниже, мы дадим более формальное определение.

Конфигурачией над $N$ мы называем пару $(L, \pi)$, где $L-$ конечное множество, а $\pi: L \rightarrow N$ - отображение. Две конфигурации $(L, \pi)$ и $\left(L^{\prime}, \pi^{\prime}\right)$ над $N$ мы назовем совпадающими, если существует биекция $\theta: L \rightarrow L^{\prime}$, такая, что $\pi=\pi^{\prime} \circ \theta$.

Рассмотрим конфигурацию $(L, \pi)$ над $N$. Для любого $a \in N$ мы обозначим через $l_{a}$ число точек, лежащих в прообразе точки $a$. Это число мы будем называть кратностью точки $а$ в конфигурации $(L, \pi)$. Очевидно конфигурации совпадают, если совпадают кратности всех точек множества $N$. Таким образом, мы возвращаемся к определению конфигурации, данному в начале пункта.

Пусть $(L, \pi)$ - конфигурация над $N$. Связкой на $(L, \pi)$ мы назовем разбиение множества $L$ на двухточечные и одноточечные подмножества:

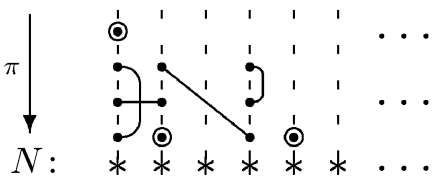

На рисунке элементы множества $N$ обозначены звездочками, элементы $L$ жирными точками. Одноточечные множества обведены кружочками, элементы двухточечных подмножеств соединены дугами.

Множество всех связок на конфигурации $(L, \pi)$ мы обозначим через $\operatorname{tng}(L, \pi)$. Множество всех одноточечных подмножеств связки $S \in \operatorname{tng}(L, \pi)$ 
мы обозначим через оne $(S)$, а множество двухточечных подмножеств - чеpe3 two $(S)$.

2.6. Другая формула для гауссовых векторов. Обозначим через $\mathscr{X}$ множество всех переменных $x_{1}, x_{2}, \ldots$ Обозначим через $\operatorname{Conf}(\mathscr{X})$ множество всех конфигураций над $\mathscr{X}$. Теперь теорема 2 может быть переформулирована в следующем виде:

Teорема 2'. Гауссову вектору $b[Q \mid r] \in F\left(l_{2}(\omega)\right)$ соответствует әлемент $\mathfrak{B}^{K}[Q \mid r]$ пространства $\mathrm{Symm}_{K, \omega}$, задаваемый формулой

$$
\begin{array}{r}
\mathfrak{B}^{K}[Q \mid r](x)=\sum_{L \in \operatorname{Conf}(\mathscr{X})}\left(\prod_{i} \varkappa_{l_{i}} \sum_{S \in \operatorname{tng}(L)} \prod_{\left\{x_{\alpha_{i}}, x_{\beta_{i}}\right\} \in \operatorname{two}(S)} \widetilde{Q}\left(x_{\alpha_{i}}, x_{\beta_{i}}\right)\right. \\
\left.\times \prod_{\left\{x_{\tau_{j}}\right\} \in \operatorname{one}(S)} \tilde{r}\left(x_{\tau_{j}}\right)\right),
\end{array}
$$

где через $l_{i}$ обозначена кратность переменной $x_{i}$ в конфигурачии $L$.

ДокАЗАТЕльСтво. Нужно убедиться, что коэффициенты разложения правой части формулы (22) по функциям $m_{\lambda}$ совпадают с приведенными в формуле (26) (см. ниже). Равносильность теоремы 2 и теоремы $2^{\prime}$ вытекает из формулы (25).

2.7. Функции $\mathfrak{R}[Q \mid r]$. Пусть $Q=Q(n, \tilde{n})=q_{n \tilde{n}}$ - функция на $N \times N$, такая, что $Q(n, \tilde{n})=Q(\tilde{n}, n)$. Пусть $r=r(n)=r_{n}$ - функция на $N$. Определим функцию $\mathfrak{R}[Q \mid r]$ на множестве конфигураций по формуле

$$
\begin{aligned}
\mathfrak{R}[Q \mid r](L, \pi) & =\mathfrak{R}[Q \mid r]\left(l_{1}, l_{2}, \ldots\right) \\
& =\sum_{S \in \operatorname{tng}(L, \pi)}\left(\prod_{\left\{\sigma_{i}, \delta_{i}\right\} \in \operatorname{two}(S)} Q\left(\sigma_{i}, \delta_{i}\right) \cdot \prod_{\left\{\tau_{\alpha}\right\} \in \operatorname{one}(S)} r\left(\tau_{\alpha}\right)\right),
\end{aligned}
$$

где через $l_{n}$ обозначена кратность точки $n \in N$ в конфигурации $(L, \pi)$.

Дадим два других определения величин $\mathfrak{R}[Q \mid r]\left(l_{1}, l_{2}, \ldots\right)$. Во-первых,

$$
\exp \left(\frac{1}{2} \sum q_{i j} z_{i} z_{j}+\sum r_{j} z_{j}\right)=\sum_{l_{1}, l_{2}, \ldots} \mathfrak{R}[Q \mid r]\left(l_{1}, l_{2}, \ldots\right) \prod_{j} \frac{z_{j}^{l_{j}}}{l_{j} !} .
$$

Во-вторых,

$$
\frac{1}{\prod l_{j} !} \mathfrak{R}[Q \mid r]\left(l_{1}, l_{2}, \ldots\right)=\sum\left\{\prod_{i>j} \frac{q_{i j}^{s_{i j}}}{s_{i j} !} \prod_{i} \frac{q_{i i}^{s_{i i}}}{2^{s_{i i}} s_{i i} !} \prod_{i} \frac{r_{i}^{t_{i}}}{t_{i} !}\right\},
$$

где суммирование ведется по всем наборам неотрицательных чисел $s_{i j}, r_{i}$, таким, что $s_{i j}=s_{j i}$ и для всех $j$ выполнено

$$
r_{j}+\sum_{i \neq j} s_{i j}+2 s_{j j}=l_{j} .
$$

2.8. Разложение гауссовых векторов по базисам $\mathfrak{q}_{n_{1}, n_{2}, \ldots}$. Рассмотрим два формальных ряда $K(h)=\sum \varkappa_{j} h^{j}, M(h)=\sum \mu_{j} h^{j}$, где $\varkappa_{0}=\mu_{0}=1$, 
$\varkappa_{1} \neq 0, \mu_{1} \neq 0$. Рассмотрим также последовательность $\omega$, от которой сейчас ничего не будет зависеть. Рассмотрим гауссов вектор $b[Q \mid r] \in F\left(l_{2}(\omega)\right)$ и его образ $\mathfrak{B}^{M}[Q \mid r](x)$ в $\operatorname{Symm}_{M, \omega}$. Нас интересует, как разложить функцию $\mathfrak{B}^{M}[Q \mid r](x)$ по базису $\mathfrak{q}_{n_{1}, n_{2}, \ldots}^{K}$. Для этого рассмотрим диаграмму Юнга $\Sigma=\left(1^{n_{1}} 2^{n_{2}} \ldots\right)$, т.е. диаграмму, у которой ровно $n_{j}$ строк длины $j$ (для всех $j$ ). Назовем строчныл разрезанием разрезание диаграммы Юнга на горизонтальные полоски вида, указанного на рисунке

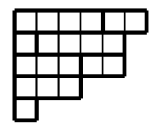

Иными словами, взять строчное разрезание - значит взять упорядоченное разбиение для каждой строки диаграммы.

Каждому куску строчного разрезания мы поставим в соответствие натуральное число, равное его длине. Тогда мы можем рассматривать множество кусков строчного разрезания $T$ как конфигурацию над $\mathbb{N}$. Обозначим эту конфигурацию через $\mathscr{W}_{T}$. Далее для данного строчного разрезания $T$ через $\zeta_{s}=\zeta_{s}(T)$ мы обозначим число кусков, на которые разрезана строка с номером $s$.

TЕОРема 3. Пусть $M(h)=K\left(\sum_{j>0} \sigma_{j} h^{j}\right)$. Тогдa

$$
\mathfrak{B}^{M}[Q \mid r]=\sum_{n_{1}, n_{2}, \ldots} A_{n_{1}, n_{2}, \ldots} \mathfrak{q}_{n_{1}, n_{2}, \ldots}^{K},
$$

где коэффичиенты $A_{n_{1}, n_{2}, \ldots}$ вычисляются по формуле

$$
A_{n_{1}, n_{2}, \ldots}=\sum_{T}\left(\prod_{s} \sigma_{\zeta_{s}} \cdot \mathfrak{R}[Q \mid r]\left(\mathscr{W}_{T}\right)\right) .
$$

В этой формуле суммирование ведется по всем строчньл разрезаниям диаграммь $\Sigma=\left(1^{n_{1}} 2^{n_{2}} \ldots\right)$, произведение берется по всем строкам диаграммьи, а функиии $\mathfrak{R}$ определены в предыдущем пункте.

ДокАЗАТЕЛЬСТво состоит в непосредственном вычислении выражения $\mathfrak{B}^{M}[Q \mid r](x)=\exp \left\{\sum q_{i j} \frac{\partial^{2}}{\partial \alpha_{i} \partial \alpha_{j}}+\sum r_{j} \frac{\partial}{\partial \alpha_{j}}\right\} \cdot \prod_{\xi} K\left(\sum_{n>0} \sigma_{n}\left(\sum_{j>0} \alpha_{j} x_{\xi}^{j}\right)^{n}\right)$.

Множитель $\exp \{\}$ мы расписываем с помощью формулы (24) и далее непосредственно вычисляем производные, при этом многочисленные факториалы благополучно сокрашаются.

ЗАмЕчАниЕ. В частности, мы получаем формулы для разложения функций $\mathfrak{B}^{K}$ по мономиальным симметрическим функциям $m_{\lambda}$, а также по функциям $p_{1}^{k_{1}} p_{2}^{k_{2}} \ldots$ (что имеет смысл в случае $\left.K(h)=\exp (h), K(h)=1+h\right)$. Забавно, что разложения по другим стандартным базисам $\left(e_{\lambda}, h_{\lambda}\right.$, функциям Шура, см. [3]) имеют вид, очень похожий на (26) (мы опускаем эти формулы). 
ЗАмечАниЕ. Рассмотрим два изоморфизма $I_{1}: F\left(l_{2}(\omega)\right) \rightarrow \operatorname{Symm}_{M, \omega}$ и $I_{2}: F\left(l_{2}(\nu)\right) \rightarrow \operatorname{Symm}_{K, \nu}$. Тогда

$$
\left(I_{2}^{-1} I_{1} b[Q \mid r]\right)(z)=\sum A_{n_{1}, n_{2}, \ldots} \frac{z_{1}^{n_{1}} z_{2}^{n_{2}} \cdots}{n_{1} ! n_{2} ! \ldots}
$$

\section{§3. Пространство кососимметрических функций}

3.1. Фермионное пространство Фока. Пусть .., $\xi_{-1}, \xi_{0}, \xi_{1}, \xi_{2}, \ldots$ - набор антикоммутирующих переменных (т.е. $\xi_{i} \xi_{j}=-\xi_{j} \xi_{i}$ ). Рассмотрим пространство $\Lambda$, базис которого образуют всевозможные одночлены вида

$$
\xi_{i_{0}} \xi_{i_{1}} \xi_{i_{2}} \xi_{i_{3}} \ldots,
$$

где $i_{0}>i_{1}>i_{2}>\ldots$ и $i_{k}=-k$ для достаточно больших $k$. Пространство $\Lambda$ - одна из версий фермионного пространства Фока (если я не ошибаюсь, эта версия впервые упоминается в [1]), см. [5, 4.1].

Скалярное произведение в $\Lambda$ задается условием, что векторы (27) образуют ортонормированный базис.

3.2. Стандартный переполненный базис. Неформально говоря, стандартный переполненный базис в $\Lambda$ состоит из произведений вида $l_{1}(\xi) l_{2}(\xi) \ldots$, где $l_{j}(\xi)$ - линейные формы. Так как такие произведения имеют право расходиться, мы должны сформулировать это аккуратнее.

Первый способ. Пусть $C=\left\{c_{i j}\right\}, i=1,2, \ldots, j=0,1,2, \ldots$, - гильбертшмидтовская матрица (т. е. $\operatorname{tr} C^{*} C<\infty$ ). Мы положим

$$
\Xi(C)=\prod_{j=\infty}^{0}\left(\xi_{-j}+c_{j 1} \xi_{1}+c_{j 2} \xi_{2}+\ldots\right):=\left(\xi_{0}+\sum_{k>0} c_{0 k} \xi_{k}\right)\left(\xi_{-1}+\sum_{k>0} c_{1 k} \xi_{k}\right) \ldots
$$

Тогда

$$
\langle\Xi(C), \Xi(D)\rangle_{\Lambda}=\operatorname{det}\left(1+C D^{*}\right)
$$

(и, в частности, норма $\|\Xi(C)\|_{\Lambda}$ конечна).

Второй способ. Пусть $R=\left\{r_{i j}\right\}$ - матрица, причем $i$ пробегает числа $0,-1,-2, \ldots$, а $j$ пробегает $\mathbb{Z}$ и при этом $r_{i j}=0$ при $j<i$. Пусть для достаточно больших по модулю $i$ выполнено равенство $r_{i i}=1$. Тогда положим

$$
\Xi\{R\}=\prod_{i=-\infty}^{0}\left(\sum_{j=i}^{\infty} r_{i j} \xi_{j}\right)
$$

При формальном раскрытии скобок в этом выражении возник бы континуум членов. Мы, однако, потребуем, чтобы из всех скобок, кроме конечного числа, бралось первое слагаемое.

Выражение (29) во всяком случае имеет смысл как формальный ряд по базису (27), однако может оказаться, что норма $\|\Xi(R)\|_{\Lambda}$ бесконечна.

ЗАмечАниЕ. Выражение (28) имеет вид (29); более того (28) представимо в виде (29) многими разными способами. В то же время выражение (27), имеющее форму (29), в виде (28) не представимо. 
3.3. Пространство кососимметрических функций. Рассмотрим формальный символ $\infty$. Назовем псевдоодночленом выражение вида

$$
x_{0}^{\infty+a_{0}} x_{1}^{\infty+a_{1}} \ldots,
$$

причем мы потребуем, чтобы $a_{j}=-j$ для достаточно больших $j$. Псевдомногочленом мы назовем формальную (вообще говоря, бесконечную) линейную комбинацию псевдоодночленов.

Назовем кососимметрическим формальным рядом псевдомногочлен, кососимметричный относительно группы $S_{\infty}$ всех финитных перестановок переменных $x_{1}, x_{2}, \ldots$ Пространство всех кососимметрических формальных рядов мы обозначим через $\widehat{\mathrm{ASymm}}$.

Рассмотрим кососимметрические формальные ряды

$$
S_{\alpha_{0}, \alpha_{1}, \ldots}:=\sum_{\sigma \in S_{\infty}}(-1)^{\sigma} x_{\sigma(0)}^{\infty+\alpha_{0}} x_{\sigma(1)}^{\infty+\alpha_{1}} \cdots
$$

(где $\alpha_{j}=-j$ для больших $j$ ). Конечные линейные комбинации формальных рядов $S_{\alpha_{0}, \alpha_{1}, \ldots}$ мы назовем кососимметрическими многочленами. Пространство всех кососимметрических многочленов мы обозначим через ASymm. Скалярное произведение в ASymm мы определим условием, что многочлены $S_{\alpha_{0}, \alpha_{1}, \ldots}$ образуют ортонормированный базис в ASymm.

ЗАмечаниЕ. Обозначим через $\mathrm{ASymm}_{n}$ пространство многочленов от $n$ переменных, кососимметричных относительно перестановок переменных. Любой такой многочлен имеет вид

$$
g\left(x_{0}, \ldots, x_{n-1}\right) \cdot \prod_{0<i<j \leqslant n-1}\left(x_{i}-x_{j}\right),
$$

где $g\left(x_{0}, \ldots, x_{n-1}\right)$ - симметрический многочлен. Каноническую проекцию $\pi_{n+1}: \mathrm{ASymm}_{n+1} \rightarrow \mathrm{ASymm}_{n}$ мы определим по формуле

$$
\left(\pi_{n+1} f\right)\left(x_{0}, \ldots, x_{n-1}\right)=f\left(x_{0}, \ldots, x_{n-1}, 0\right) \cdot\left(x_{0} x_{1} \ldots x_{n-1}\right)^{-1} .
$$

Тогда ASymm есть обратный предел пространств $\mathrm{ASymm}_{n}$ в категории $\mathbb{Z}$-градуированных пространств (мы полагаем, что степень однородности ряда $S_{\alpha_{0}, \alpha_{1}, \ldots}$ равна $\left.\sum_{j}\left(\alpha_{j}+j\right)\right)$.

ЗАмЕчАНИЕ. Пусть $f(x)$ — кососимметрический формальный ряд. Тогда

$$
f(x)=\left(x_{0}^{\infty} x_{1}^{\infty-1} x_{2}^{\infty-2} \ldots\right) g(x),
$$

где $g(x)$ - обычный формальный ряд по положительным и отрицательным степеням $x_{i}$ (т.е. бесконечная линейная комбинация одночленов вида $x_{0}^{\beta_{0}} x_{1}^{\beta_{1}} \ldots x_{s}^{\beta_{s}}$, где $\left.\beta_{j} \in \mathbb{Z}\right)$. Пусть $P_{i j} g(x)$ - выражение, полученное из $g(x)$ перестановкой $x_{i}$ и $x_{j}$ (где $i>j$ ). Тогда

$$
P_{i j} g(x)=-\frac{x_{i}}{x_{j}} g(x),
$$

и, обратно, если $g(x)$ удовлетворяет этому условию, то выражение (31) является кососимметрическим рядом. 
3.4. Фермион-кососимметрическое соответствие. Естественный изоморфизм между пространством кососимметрических функций и фермионным пространством Фока определяется так: вектору $\xi_{\alpha_{0}} \xi_{\alpha_{1}} \ldots$ ставится в соответствие кососимметрическая функция $S_{\alpha_{0}, \alpha_{1}, \ldots}$, заданная формулой $(30)$.

3.5. Переполненный базис в пространстве кососимметрических функций. Рассмотрим систему функций

$$
q_{j}(x)=\sum_{k \geqslant 0} a_{k}^{(j)} x^{k}, \quad j=0,1,2, \ldots,
$$

и пусть при этом $a_{0}^{(j)}=1$ при достаточно больших $j$. Рассмотрим кососимметрическую функцию

$$
\begin{aligned}
& \Xi\left[q_{0}, q_{1}, \ldots\right]
\end{aligned}
$$

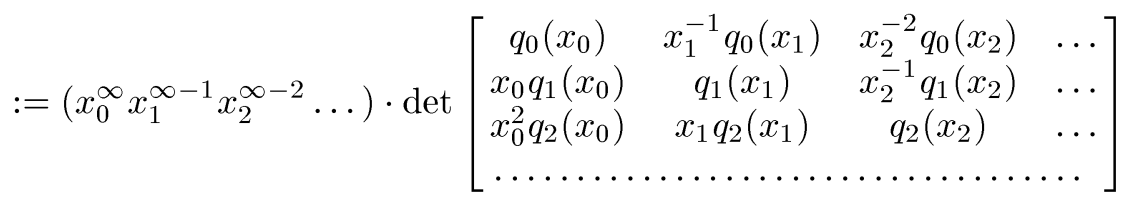

(из этого определителя берутся произведения, отличающиеся лишь конечным числом сомножителей от $\left.q_{1}\left(x_{0}\right) q_{2}\left(x_{1}\right) \ldots\right)$.

Легко видеть, что кососимметрическая функция $\Xi\left[q_{1}, q_{2}, \ldots\right]$ соответствует вектору $\Xi\{R\} \in \Lambda$, заданному формулой $(29)$, где $r_{i j}=a_{j-i+1}^{(-i+1)}$.

3.6. Соответствие между кососимметрическими и симметрическими функциями. Через $\Delta$ мы обозначим кососимметрическую функцию

$$
S_{0,-1,-2, \ldots}=\sum_{\sigma \in S_{\infty}}(-1)^{\sigma} x_{\sigma(0)}^{\infty} x_{\sigma(1)}^{\infty-1} x_{\sigma(2)}^{\infty-2} \cdots=\prod_{j \geqslant 0} x_{j}^{\infty-j} \prod_{\alpha<\beta}\left(1-\frac{x_{\beta}}{x_{\alpha}}\right) .
$$

Удобно думать или лучше положить по определению (это будет своего рода нормировка символа $\infty)$, что

$$
\Delta=\prod_{0 \leqslant i<j<\infty}\left(x_{i}-x_{j}\right)
$$

Пусть $f$ - симметрическая функция. Тогда $f \cdot \Delta$ есть кососимметрическая функция, причем оператор $f \mapsto f \cdot \Delta$ является унитарным оператором из пространства Symm симметрических функций (зависящих от $\left.x_{0}, x_{1}, \ldots\right)$, снабженного классическим скалярньл произведением, в пространство ASymm кососимметрических функций.

ЗАмЕЧАНИЕ. Оператор $f \mapsto f \cdot \Delta$ есть не что иное, как бозон-фермионное соответствие (см. [8]).

3.7. Примеры. а) Функции $S_{\alpha_{1}, \alpha_{2} \ldots} \in$ ASymm соответствуют функциям Шура (см. [3]) в Symm (см. [8]).

б) Бозонный переполненный базис. Пусть $q(t)=1+\sum_{k>0} a_{k} t^{k}$. Рассмотрим симметрическую функцию $\prod_{j=0}^{\infty} q\left(x_{j}\right)$. Легко видеть, что соответствующая ей кососимметрическая функция равна $\Xi[q, q, \ldots]$, а соответствующий 
ей элемент фермионного пространства Фока записывается в виде $\Xi(C(q))$ (см. (28)), где матричные элементы $c_{i j}(q)$ матрицы $C(q)$ определяются из соотношения

$$
\sum_{i, j \geqslant 0} c_{i j}(q) z^{i} u^{j}=\frac{q(z) / q(u)-1}{z-u} .
$$

в) Образы Фермионных векторов в бозонном пространстве. Пусть $c(\xi) \in \Lambda$. Тогда в силу (5) соответствующий элемент $g \in F$ задается формулой

$$
g\left(z_{1}, z_{2}, \ldots\right)=\left\langle c(\xi), \Xi\left[C\left(q_{z}\right)\right]\right\rangle_{\Lambda},
$$

где

$$
q_{z}(t)=\exp \left(\sum \frac{z_{j} t^{j}}{j}\right)
$$

В частности, вектору $\Xi(A)$ соответствует функция $\operatorname{det}\left(1+A C\left(q_{z}\right)\right)$.

г) Алгебра Bирасоро. Пусть $\theta$ - та же функция, что и в п. 1.7. Тогда в обозначениях (32)

$$
\Delta \cdot \prod_{i>j}\left(\frac{\theta\left(x_{i}\right)-\theta\left(x_{j}\right)}{x_{i}-x_{j}}\right)=\prod_{i>j}\left(\theta\left(x_{i}\right)-\theta\left(x_{j}\right)\right)=\Xi\left[\theta, \theta^{2}, \theta^{3}, \ldots\right] .
$$

\section{ЛитеРАТУРА}

1. Березин Ф. А. Несколько замечаний о представлениях соотношений коммутации. УМН, 24, вып. 4, 65-88 (1969).

2. Kerov $S$. V. Generalized Hall-Littlewood symmetric functions and orthogonal polynomials. Adv. Soviet Math., 9, 67-94 (1992).

3. Macdonald I. G. Symmetric functions and Hall polynomials. Second edition, Clarendon Press, Oxford, 1995. (Имеется перевод первого издания 1979 г.: Макдональд И. Симметрические функции и многочлены Холла. Мир, М., 1985.)

4. Неретин Ю. А. Голоморфные продолжения представлений группы диффеоморфизмов окружности со старшим весом. Матем. сб., 180, № 5, 635-657 (1989).

5. Neretin Yu. A. Categories of symmetries and infinite dimensional groups. Clarendon Press, Oxford, 1996.

6. Неретин Ю. А. О соответствии между бозонным пространством Фока и пространством $L^{2}$ по мере Пуассона. Матем. сб., 188, № 11, 1997.

7. Odzijevicz A. Quantum algebras and $q$-special function related to coherent states on the disc. Preprint, Warsaw University.

8. Прессли А., Сигал Г. Группы петель. Мир, М., 1990.

Московский институт электроники и математики Max-Planck-Institut für Mathematik, Bonn
Поступила в редакцию 18 июня 1997 г. 